\title{
NOTIZIA BIOGRAFICA
}

1882: Paul Friedländer nasce a Berlino il 21 marzo. Il padre è uno spedizioniere di confessione evangelica.

1900: Dopo aver terminato le scuole a Berlino inizia a frequentare i corsi di Filologia Classica e Archeologia presso le Università di Berlino e Bonn, dove ha per maestri Hermann Usener e Ulrich von WilamowitzMöllendorff. Si laurea nel 1905. Dopo la laurea compie lunghi viaggi in Asia minore, Grecia e Italia.

1907-8: Usufruisce di una borsa di studio presso il Kaiserlichen Deutschen Archäologischen Institut di Berlino.

1909: Ottiene un posto di Oberlebrer presso l'Humboldtgymnasium di Berlino.

1911: Consegue l'abilitazione all'insegnamento presso l'Università di Berlino.

1914-15: Diventa professore straordinario.

1915-18: Entra volontario nell'esercito con il grado di tenente. Prende parte direttamente a numerose azioni belliche ed è insignito della croce di ferro di seconda classe.

1920: Professore ordinario all'Università di Marburgo.

1932: Chiamato a insegnare presso l'Università di Halle. 
1935: Rimosso dall'incarico perché di origini ebraiche, è rinchiuso per sei settimane a Sachsenhausen. Liberato per intercessione di Rudolf Bultmann, dopo un breve periodo trascorso a Roma come bibliotecario della Gregoriana e della Vaticana, deve di nuovo emigrare per l'estensione all'Italia delle leggi antisemite. Nel 1939 raggiunge con la famiglia gli Stati Uniti, dove inizia ad insegnare presso la John Hopkins University di Baltimora.

1940-9: Si trasferisce all'University of California di Los Angeles, dove nel 1945 ottiene l'ordinariato in filologia classica e nel 1949 la carica onorifica di faculty research lecturer.

1968: Professore emerito di filologia classica, muore il 12 dicembre, a ottantasei anni. 